\title{
AA Amyloidosis in a Cohort of 128 Patients with Takayasu's Arteritis
}

\author{
Nikolay Mukhin ${ }^{1}$, Ilya Smitienko ${ }^{2}$, Pavel Novikov ${ }^{1}$, Sergey Moiseev $^{1^{*}}$ and Tatiana Shevtsova ${ }^{1}$ \\ ${ }^{1}$ Clinic of Nephrology, Internal and Occupational Diseases, Sechenov First State Medical University, Moscow \\ 'Peoples' Friendship University, Moscow, Russia
}

*Corresponding author: S Moiseev, Clinic of Nephrology, Internal and Occupational Diseases, First Moscow State Medical University, Rossolimo, 11/5, Moscow 119435; Tel: 749924825 44; Fax: 790159044 91; Email: clinpharm@mtu-net.ru

Received date: Dec 16, 2016; Accepted date: Feb 8, 2017; Published date: Feb 17, 2017

Copyright: (c) 2017 Mukhin N. This is an open-access article distributed under the terms of the Creative Commons Attribution License, which permits unrestricted use, distribution, and reproduction in any medium, provided the original author and source are credited.

\begin{abstract}
Systemic AA amyloidosis is a relatively rare complication of chronic inflammatory disorders, e.g. rheumatoid arthritis, ankylosing spondylitis, familial Mediterranean fever and other autoinflammatory syndromes, Crohn's disease, malignancies and recurrent infections. Isolated case of AA amyloidosis have been reported in many other chronic diseases, including systemic vasculitides, diffuse connective tissue diseases, sarcoidosis, chronic hepatitis, etc. In the past ten years the incidence of AA amyloidosis decreased significantly due to earlier and more aggressive treatment of underlying conditions such as rheumatoid arthritis or infections. In the recent series of 474 patients with renal amyloidosis from Mayo clinic there were only $7 \%$ cases of AA amyloidosis while $86 \%$ patients presented with $\mathrm{AL}$ form of disease. Nevertheless, AA amyloidosis remains a life-threatening complication of immune-mediated and inflammatory conditions and deserves effective prevention and treatment. Takayasu's arteritis (TAK) is a rare largevessel vasculitis of unknown etiology that more frequently affects women of childbearing age and is defined as "granulomatous inflammation of the aorta and its major branches" by the revised Chapel Hill Consensus Conference. The search in Pubmed database revealed only a dozen reports of AA amyloidosis in patients with TAK. The objective of our study was to evaluate the incidence of AA amyloidosis in a cohort of consecutive TAK patients.
\end{abstract}

Keywords: Amyloidosis; Arteries; Hematuria; Proteinuria

\section{Objective}

To evaluate the incidence of AA amyloidosis in a cohort of consecutive TAK patients.

\section{Patients and Methods}

We retrospectively studied medical records of 128 consecutive patients with TAK who met the American College of Rheumatology classification criteria. The diagnosis of amyloidosis that was suspected clinically (new proteinuria or nephrotic syndrome) was confirmed histologically by the presence of congophilic fibrillar inclusions.

\section{Methods}

We retrospectively studied medical records of 128 consecutive patients with TAK who were evaluated from 1979 through 2012 in our clinic that serves as a tertiary center for systemic vasculitides in Russia. All patients met the American College of Rheumatology classification criteria for TAK [1] and the definition of the 2012 revised International Chapel Hill Consensus Conference Nomenclature of Vasculitides [2]. Imaging studies were available in all patients and included ultrasonography $(n=118)$, conventional angiography $(n=31)$, computed tomographic angiography $(\mathrm{n}=7)$ and positron emission tomography $(n=32)$.

The diagnosis of amyloidosis that was suspected clinically (new proteinuria or nephrotic syndrome) was confirmed histologically by the presence of congophilic fibrillar inclusions. Amyloidosis type AA was established by immunohistochemistry and by exclusion of other types of amyloidosis, e.g. light chain amyloidosis.

\section{Patient 1:}

A 27-year old male presented with a 16-year history of recurrent fever, arthritis, anemia, persistent increase in ESR (up to $64 \mathrm{~mm} / \mathrm{h}$ ) and C-reactive protein (CRP). These episodes were treated effectively with low dose corticosteroids. One year ago ultrasound examination showed stenosis of both carotid arteries and abdominal aorta. Type 5 TAK was diagnosed. The patient was treated with prednisolone at a daily dose of $30 \mathrm{mg}$ and azathioprine $150 \mathrm{mg}$ daily. There were also mild proteinuria, microhematuria, increased TTH and atrophic changes of thyroid gland on ultrasound examination. Thyroid morphology with Congo red staining revealed amyloid deposits in the gland stroma. Rectal biopsy confirmed diagnosis of AA amyloidosis. In the following two years the patient presented with subnephrotic proteinuria and stable renal function.

\section{Patient 2:}

A 25-year old female presented with arterial hypertension, recurrent low grade fever, anemia, fatigue and increased ESR (up to $76 \mathrm{~mm} / \mathrm{h}$ ) and CRP for 3 years. A year ago she complained of neck pain in the region of the carotid bifurcation. Systolic bruit was found over the carotid arteries. On ultrasound examination there was stenosis of right carotid artery, vertebral arteries and abdominal aorta that was compatible with type 5 TAK. She was treated with prednisolone $30 \mathrm{mg}$ daily. Clinical symptoms have improved partially while laboratory signs of inflammation have persisted. Prior to admission to the clinic proteinuria and hematuria were detected. She also complained of abdominal pain and constipation. Colonoscopy was unremarkable but histologic study of colonic mucosa with Congo red staining showed amyloid deposits. Immunosuppressive treatment during the next 15 years was associated with stable vascular disease and no worsening of kidney function. 


\section{Patient 3:}

A 45 -year old female had a 20 -year history of recurrent low grade fever, pain in the neck and arthralgia. Laboratory tests showed high ESR (up to $70 \mathrm{~mm} / \mathrm{h}$ ) and anemia (hemoglobin $46 \mathrm{~g} / \mathrm{L}$ ). Diagnosis was unclear but for 16 years she received maintenance treatment with prednisolone at a daily dose of up to $30 \mathrm{mg}$. The attempts to taper the dose of corticosteroids were associated with relapses of the disease. At the age of 44 years a systolic blood pressure difference of 30-40 mm Hg between two arms was found. Clinical investigation showed high ESR $(77 \mathrm{~mm} / \mathrm{h})$, proteinuria (1.2 g daily), Contrast angiography revealed stenosis of celiac trunk and both renal arteries while positron emission tomography (PET) with 18-fluorodeoxyglucose showed generalised involvement of arterial tree (including both carotid arteries). Type 5 TAK was diagnosed. Rectum and gastric biopsy with Congo red staining revealed amyloid deposits. AA amyloThree stents were successfully implanted in the ostium of the celiac trunk and both renal arteries. She was also treated with corticosteroids and methotrexate. Clinical symptoms improved after increase in prednisolone dose up to $40 \mathrm{mg}$ daily but proteinuria worsened to nephrotic syndrome, and she developed microhematuria. There was progression of nephrotic syndrome with development of kidney failure. The patient died from pneumonia and sepsis.

\section{Patient 4:}

A 27-year old female presented with recurrent fever, pain in the neck, anemia and high ESR (up to $55 \mathrm{~mm} / \mathrm{h}$ ) for 2 years. Ultrasound examination showed stenosis of both carotid arteries and asymmetic narrowing of subclavian arteries. Type 1 TAK was diagnosed and she was treated with hydroxychloroquine and NSAIDs with incomplete effect. One year later she developed proteinuria and increased serum creatinine level. Rectal biopsy with Congo red staining confirmed diagnosis of AA amyloidosis. The patient died suddenly from pulmonary embolism.

\section{Patient 5:}

A 33-year old women presented with a 7-year history of low grade fever, headache, dizziness and tenderness in the neck that were treated symptomatically with NSAIDs. At the age of 29 years examination revealed anemia (hemoglobin $57 \mathrm{~g} / \mathrm{L}$ ), increased ESR (up to $72 \mathrm{~mm} / \mathrm{h}$ ) and a systolic blood pressure difference of $30 \mathrm{~mm} \mathrm{Hg}$ between two arms. Ultrasound showed stenosis of both subclavian and carotid arteries. Type 3 TAK was diagnosed. She was treated with prednisolone $40 \mathrm{mg}$ daily and colchicine with incomplete effect. Within the next 3 years she developed proteinuria 1-2 g daily and microhematuia. Rectal biopsy with Congo red staining showed amyloid deposits. Adequate immunosuppressive treatment induced remission of systemic vasculitis. At 4 years of follow-up she presented with nephrotic syndrome and stable renal function.

In the group of patients with AA amyloidosis there were 4 females and 1 male. The median duration of TAK prior to development of amyloidosis was 13 years (from 3 to 20 ). Three patients (60\%) had generalized (type 5) arteries involvement (vs. $38 \%$ in the whole cohort). All patients recevied immunosuppressive treatment (corticosteroids at medium doses in 5 of them) but were unable to achieve sustained remission of TAK. All five patients had predominant kidney involvement (proteinuria or nephrotic syndrome). Two patients (40\%) died within a few years after diagnosis of AA amyloidosis.
Therefore, a five year survival was only $60 \%$ while in the whole cohort it reached $98 \%$.

\section{Results}

There were $20(16 \%)$ males and $108(84 \%)$ females who were followed for $5.5 \pm 5.4$ years. AA amyloidosis was diagnosed in 5 patients (4 females and 1 male) who recevied immunosuppressive treatment but were unable to achieve sustained remission (Table 1). The median duration of TAK prior to development of amyloidosis was 13 years. Three patients (60\%) had generalized (type 5) arteries involvement (vs. $38 \%$ in the whole cohort). All five patients had predominant kidney involvement (proteinuria or nephrotic syndrome). Two patients (40\%) died within a few years after diagnosis of AA amyloidosis.

\section{Discussion}

The epidemiology of the underlying diseases leading to AA amyloidosis changed significantly over the last decades due to the development of more effective treatments and better control of many diseases [3]. Chronic infections, such as tuberculosis and osteomyelitis, have long been the most common causes of AA amyloidosis while today they are more relevant only in certain developing countries [4]. The incidence of AA amyloidosis in patients with rheumatoid arthritis and ankylosing spondylitis is also decreasing as a result of earlier and more aggressive treatment with disease modifying antirheumatic drugs and biologic agents [5]. These changes may account for a relative increase in the role of other conditions, including different autoimmune disorders, as a possible cause of AA amyloidosis.

\begin{tabular}{|c|c|c|c|c|c|}
\hline Parameters & $\# 1$ & \#2 & \#3 & \#4 & \#5 \\
\hline Gender & $M$ & $\mathrm{~F}$ & $\mathrm{~F}$ & $\mathrm{~F}$ & $\mathrm{~F}$ \\
\hline $\begin{array}{l}\text { Age at TAK } \\
\text { presentation, years }\end{array}$ & 11 & 25 & 25 & 22 & 26 \\
\hline Type of TAK & 5 & 5 & 1 & 5 & 3 \\
\hline Treatment & CS & CS & $\begin{array}{l}\text { CS, } \\
\text { MTX }\end{array}$ & $\begin{array}{l}\mathrm{CS} \\
\mathrm{HCQ}\end{array}$ & $\begin{array}{l}\text { CS, } \\
\text { colchicine }\end{array}$ \\
\hline Effect & $+/-$ & $+/-$ & $+/-$ & $+/-$ & $+/-$ \\
\hline $\begin{array}{l}\text { Duration of TAK prior } \\
\text { to AA amyloidosis, } \\
\text { years }\end{array}$ & 16 & 19 & 20 & 3 & 7 \\
\hline Amyloid deposits & $\begin{array}{l}\text { Rectum, } \\
\text { thyroid }\end{array}$ & $\begin{array}{l}\text { Rectum, } \\
\text { stomach }\end{array}$ & Rectum & $\begin{array}{l}\text { Rectum, } \\
\text { bowel }\end{array}$ & Rectum \\
\hline Urine changes & Protein & NS & Protein & Protein & Protein \\
\hline Outcome & $\begin{array}{l}\text { Alive at } 2 \\
\text { years }\end{array}$ & $\begin{array}{l}\text { Alive at } \\
15 \text { years }\end{array}$ & $\begin{array}{l}\text { Death } \\
\text { (sepsis) }\end{array}$ & $\begin{array}{l}\text { Death } \\
\text { (PE) }\end{array}$ & $\begin{array}{l}\text { Alive at } 4 \\
\text { years }\end{array}$ \\
\hline
\end{tabular}

Table 1: Clinical characteristics of patients with TAK and reactive AA amyloidosis. Note: +/- incomplete effect (no sustained remission), NSnephrotic syndrome, CS-corticosteroids, MTX-methotrexate, HCQhydroxychlorochine.

Systemic vasculitides remain an uncommon ethiologic factor of AA amyloidosis. As already mentioned, there are only few isolated reports of secondary amyloidosis in TAK patients though obviously not all case reports were published. In a recent US cohort of 126 patients with 
TAK from Mayo Clinic there were no cases of AA amyloidosis at all [6]. Chinese authors also reported no cases of amyloidosis or nephrotic syndrome in a very large cohort of 566 patients with TAK who were followed for a average of $5.0 \pm 0.2$ years [7].

In our cohort of consecutive 128 patienths with TAK the prevalence of AA amyloidosis was unexpectedly high (3.9\%). All five patients had typical clinical manifestations (e.g. proteinuria and/or nephrotic syndrome) and definite biopsy-proven diagnosis of AA amyloidosis.

The factors, that could account for the development of AA amyloidosis in these particular patients, included long duration and persistent activity of systemic vasculitis and/or delayed and only partly effective treatment, e.g. no patient was able to achieve complete remission of TAK. But these factors cannot explain the difference between the rate of AA amyloidosis in our and other cohorts. For example, in a similar US cohort the median delay in diagnosis was also prolonged (17.5 months), especially in patients over 40 years of age (44.8 months) [8]. The median time to achieve sustained remission was 31.9 months, and $46 \%$ of patients developed at least one relapse at 5 years of follow-up. The approaches to treatment were also comparable in our and the US cohort, e.g. $95 \%$ and $92 \%$ of patients, respectively, were treated with corticosteroids, while $48 \%$ and $66 \%$ patients received additional immunosuppressants (usually methotrexate). Notably, the proportion of patients who required vascular interventions in our cohort was significantly lower than at Mayo Clinic (17\% vs. 55\%). But in our opinion low need in surgery or other vascular interventions, that may reflect in general less serious course of disease, should have resulted in lower incidence of AA amyloidosis [9].

It is tempting to suggest that a high incidence of AA amyloidosis in the Russian cohort of TAK patients was linked to genetic factors. But this hypothesis is speculative and we do not have any data to confirm it.

AA amyloidosis was unfavourable prognostic factor in TAK patients. At 5 years survival was only $60 \%$ while in the whole cohort it reached $98 \%$. The causes of death in two patients included infection and pulmonary embolism.

The enrolment of consecutive patients who were followed for a median of approximately 5 years (up to 15 years) was an advantage of our study. Nevertheless, we cannot rule out a selection bias as our clinic is a tertiary centre for patients both with systemic vasculitides and kidney diseases. In our opinion, this fact could not influence the results of the study as all patients with AA amyloidosis had diagnosis of TAK prior to development of kidney disease. A limitation of our study is that we did not perform kidney biopsy to confirm renal amyloidosis. But AA amyloidosis was histologically proven and other causes of kidney disease were ruled out in all patients. Notably, we did not aim to study the course and outcomes of renal amyloidosis as they are well known $[10,11]$.

\section{Conclusion}

Our study showed unexpectedly high prevalence of AA amyloidosis (3.9\%) in a cohort of 128 consecutive TAK patients. The factors that contributed to development of AA amyloidosis included generalized arteries involvement, prolonged history of disease and persistent inflammatory activity. AA amyloidosis was associated with significant worsening of survival compared with a whole cohort of TAK patients. The prevalence of AA amyloidosis in our cohort of TAK patients was unexpectedly high (3.9\%). The factors that contributed to development of AA amyloidosis included generalized arteries involvement, prolonged history of disease and persistent inflammatory activity.

\section{References}

1. Arend WP, Michel BA, Bloch DA, Hunder GG, Calabrese LH, et al. (1990) The American College of Rheumatology 1990 criteria for the classification of Takayasu arteritis. Arthritis Rheum 33: 1129-34.

2. Jennette JC, Falk RJ, Bacon PA, Basu N, Cid MC et al. (2012) Revised International Chapel Hill Consensus Conference Nomenclature of Vasculitides. Arthritis Rheum 65: 1-11.

3. Real de Asúa D, Costa R, Galván JM, Filigheddu MT, Trujillo D, et al. (2014) Systemic AA amyloidosis: epidemiology, diagnosis, and management. Clin Epidemiol 6: 369-77. Obici L, Merlini G (2012) AA amyloidosis: basic knowledge, unmet needs and future treatments. Swiss Med Wkly 142: w13580.

4. Immonen K, Finne P, Gronhagen-Riska C, Petterson T, Klaukka T et al. (2011) A marked decline in the incidence of renal replacement therapy for amyloidosis associated with inflammatory rheumatic diseases - data from nationwide registries in Finland. Amyloid 18: 25-8.

5. Schmidt J, Kermani TA, Bacani AK, Crowson CS, Cooper LT, et al. (2013) Diagnostic features, treatment, and outcomes of Takayasu arteritis in a US cohort of 126 patients. Mayo Clin Proc 88: 822-830.

6. Yang L, Zhang H, Jiang X, Zou Y, Qin F, et al. (2014) Clinical manifestations and longterm outcome for patients with Takayasu arteritis in China. J Rheumatol 41: 2439-46.

7. Said SM, Sethi S, Valeri AM, Leung N, Cornell LD, et al. (2013) Renal amyloidosis: origin and clinicopathologic correlations of 474 recent cases. Clin J Am Soc Nephrol 8: 1515-1523.

8. Nakamura S, Morishita M, Yang CL, Utsumi K, Takada H et al. (2006) An elderly female who survived more than 30 years following a diagnosis of Takayasu's arteritis, complicated by fatal intestinal amyloidosis. Clin Rheumatol 25: 907-10.

9. Jean R, Cretel E, Sicard C, Daniel L, Sichez H, et al. (2004) Amyloidosis complicating Takayasu's arteritis. Rev Med Interne 25: 399-400.

10. AteÅŸ K, Erturk S, Diker E, Karatan O, Duman N, et al. (1996) Renal amyloidosis complicating Takayasu's arteritis: a case report. S Nephron 73: 111-112.

11. Espinosa M, Rodriguez M, Martín-Malo A, Pérez R, Moreno E, et al. (1994) A case of Takayasu's arteritis, nephrotic syndrome, and systemic amyloidosis. Nephrol Dial Transplant 9: 1486-8. 